\title{
Unusual Location of a Posterior Lipoma Originating From the Left Atrial Roof: Case Report and Review of the Literature
}

\author{
Ovidiu Stiru, MD, PhD, Roxana Carmen Geana, MD, Liana Valeanu, MD, Adrian Tulin, MD, PhD, \\ Laura Raducu, MD, PhD, Vlad Anton Iliescu, MD, PhD
}

Prof. Dr. C. C. Iliescu Emergency Institute for Cardiovascular Diseases, Bucharest, Romania

\section{ABSTRACT}

Lipomas are the most common type of soft tissue tumors. They mainly are located in subcutaneous tissue in the body, including the heart. The cardiac location of lipomas is rare, mostly asymptomatic, and can cause life-threatening complications by rapid growth. The clinical symptoms, when present, occur in evolution with the growth in size, depending upon the location and degree of invasion in the endocardium.

We present the case of a 63 -year-old male patient with a large intrapericardial lipoma with an unusual location, originating from the left atrial roof. The initial symptoms of the patient were shortness of breath, dizziness, and mild dyspnea. Transthoracic echocardiography (TTE) was the first line diagnosis method, followed by computed tomography (CT); both showed a large posterior intrapericardial mass. Resection of a 12/8/5 cm lipomatous tumor mass was performed via median sternotomy, under cardiopulmonary bypass (CPB) on the beating heart. Histopathologic examination revealed the presence of diffuse proliferation of large- and medium-sized mature adipocytes consistent with the diagnosis of pericardial lipoma. The patient was discharged at home on the seventh postoperative day, with a marked improvement of his clinical state and effort tolerance. He did not present evidence of recurrence at his 1-year follow up.

\section{INTRODUCTION}

Primary tumors of the heart account for less than 0.2-0.4\% of all cardiac tumors [McAllister 1999]. The most common primary cardiac tumors in adults are myxomas [lliescu 2008], followed by papillary fibroelastomas and lipomas [McAllister 1978]. Lipomas account for $8.4 \%$ to $10 \%$ cases of primary heart neoplasms and approximately $14 \%$ of benign cardiac masses [McAllister 1978]. Since clinical findings often are nonspecific, many cardiac lipomas (CL) are found in imaging studies performed for other reasons. However, when clinical features appear, they depend on the size and tumor location.

Received February 18, 2020; accepted March 6, 2020.

Correspondence: Ovidiu Stiru, Cardiac surgery department. Prof. Dr. C. C. Iliescu Emergency Institute for Cardiovascular Diseases Sos. Fundeni 258, sector 2, 022328 Bucharest, Romania; fax: +400722207286; +400722207286 (e-mail: ovidiu_stiru@yahoo.com).
CLs are fat-containing encapsulated tumors with unknown etiology [Italiano 2008].

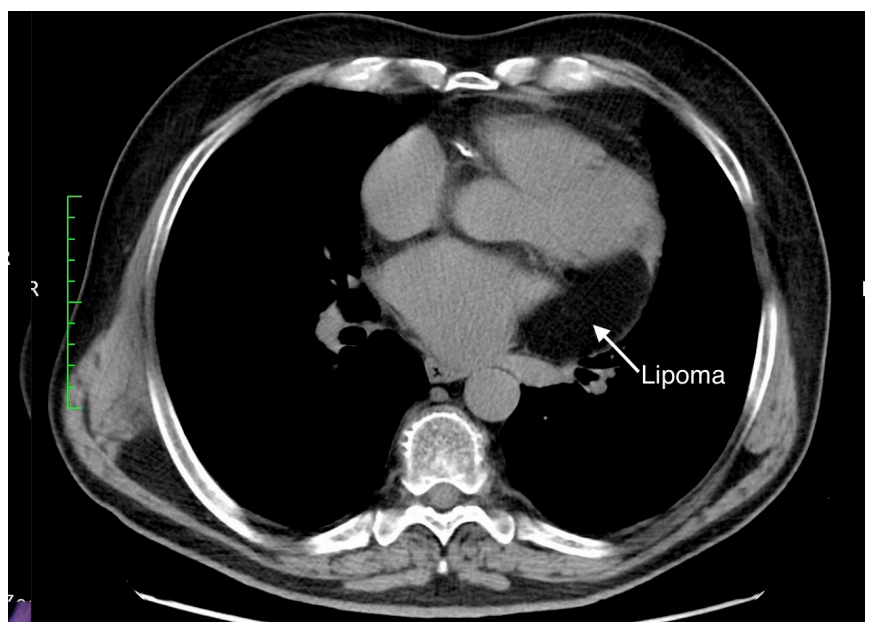

Figure 1. Thoracic CT scan. The large, ovoid tumor, $11.8 / 9.3 / 5.4 \mathrm{~cm}$ located in the posterior medi-astinum along the left border of the heart, toward the base of the heart.

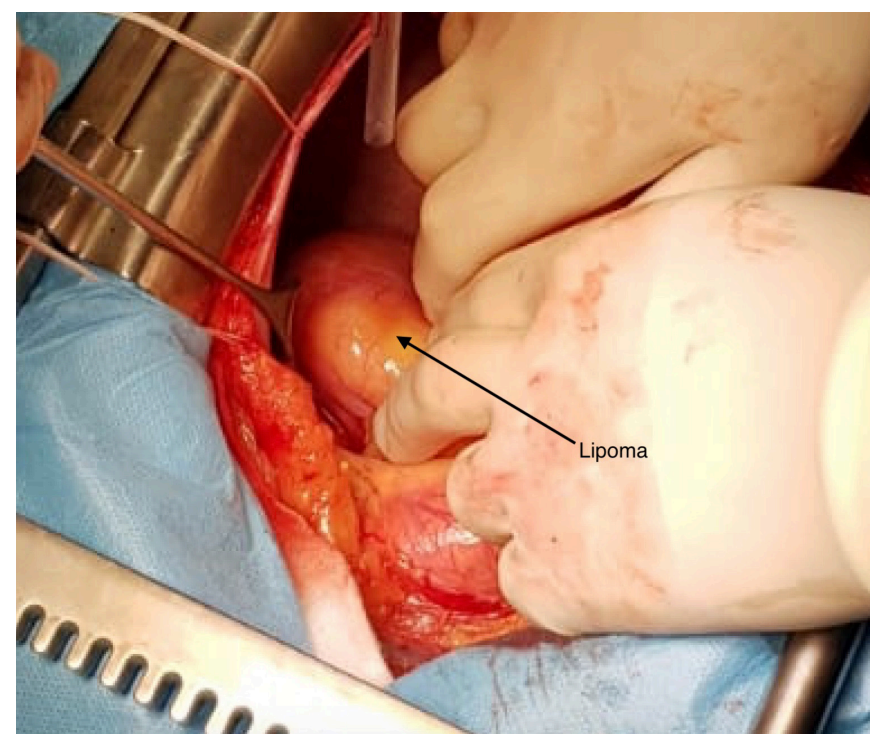

Figure 2. Intraoperative aspect-in-situ incapsulated lipomatous tumor attached to the left atrial roof. 


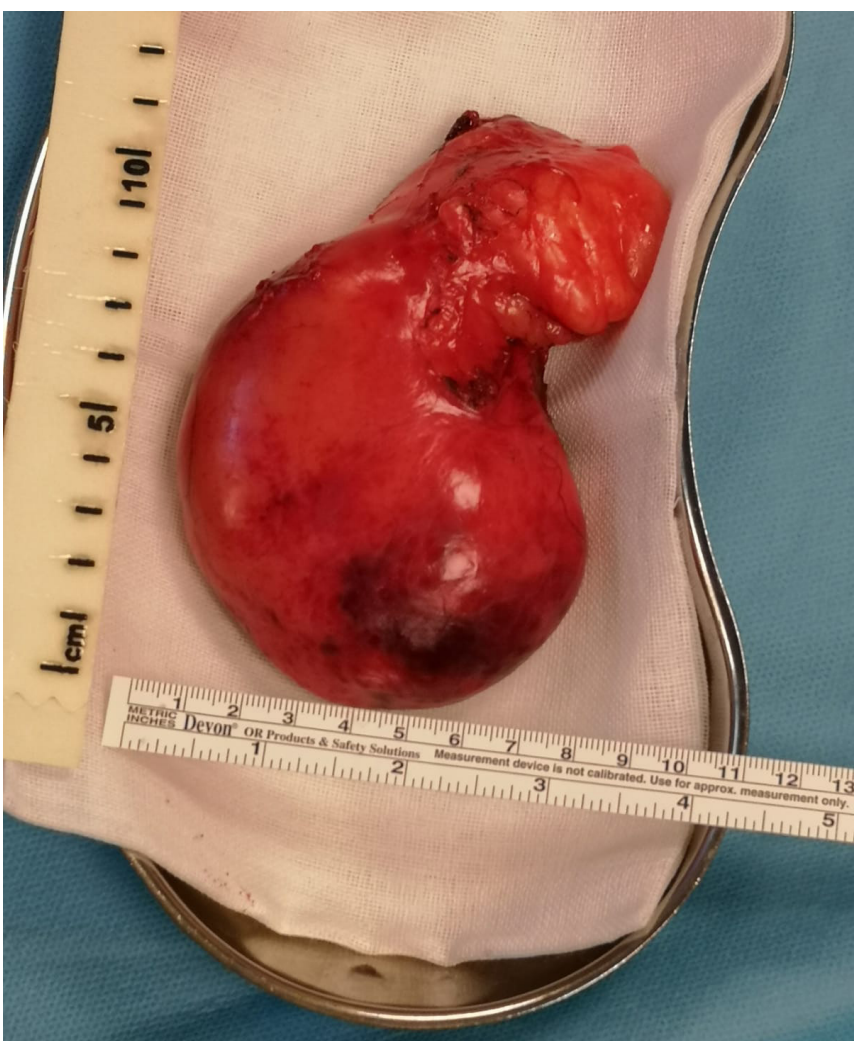

Figure 3. Postexcision-aspect of incapsulated lipomatous tumor.

\section{CASE REPORT}

A 64-year-old man was admitted to our clinic with shortness of breath that worsened over the course of a year, evolving to a NYHA class III congestive heart failure. In addition to sex and age, severe arterial hypertension, dyslipidemia, and obesity accounted for an almost complete chart of cardiovascular risk factors in this case. Other important medical and surgical history points are permanent atrial fibrillation, nephrolithiasis, 3B stage chronic kidney disease, hepatic steatosis, repaired inguinal hernia, and cholecystectomy. Despite this rather rich medical and surgical history, the overall clinical state of the patient was good. Physical examination was normal, except for faint heart sounds and irregular tachycardia $(110 \mathrm{bpm})$, without marks of lipomatosis under the skin. Electrocardiogram (ECG) showed atrial fibrillation (AF) with low amplitude QRS complexes in the frontal leads and poor $\mathrm{R}$ wave progression in precordial leads. Laboratory tests demonstrated a normal complete blood count, normal electrolytes and liver function tests, with renal dysfunction, serum creatinine 1.55 $\mathrm{mg} / \mathrm{dL}$ (local laboratory reference range 0.7 to $1.2 \mathrm{mg} / \mathrm{dL}$ ), total cholesterol $250 \mathrm{mg} / \mathrm{dL}$, LDL cholesterol $190 \mathrm{mg} / \mathrm{dL}$. The cardiothoracic ratio on a chest standard posteroanterior radiograph exceeded $50 \%$, revealing an enlargement of the cardiac size. Transthoracic echocardiography (TTE) revealed a large echolucent $48 / 76 \mathrm{~mm}$ intrapericardial mass was lying lateral and posteriorly to the left atrium (LA)

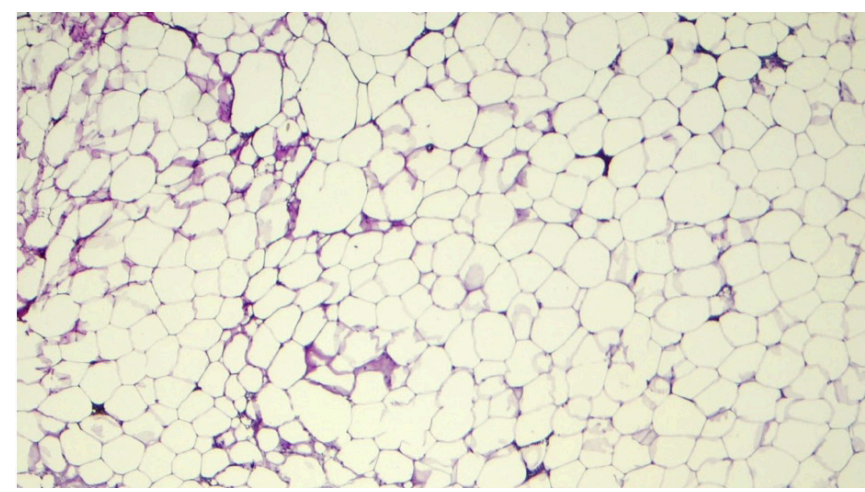

Figure 4. Histopathologic examination revealed the presence of diffuse proliferation of large and medium sized mature adipocytes consistent with the diagnosis of pericardial lipoma (H\&E staining; magnification $\times 10)$.

and left ventricle (LV). This mass exerted no compression against any of the heart chambers, at least while the patient was at rest. The contractility was normal. Following the TTE examination, a thoracic CT scan thorax confirmed the presence of an intrapericardial mass with a fat-like density, measuring $11.8 / 9.3 / 5.4 \mathrm{~cm}$ in the posterior mediastinum, located along the posterior surface of the heart from diaphragm level to pulmonary artery bifurcation level (Figure 1). In our patient, both TTE and CT failed to determine the site of origin of the tumor. Cardiothoracic surgery was consulted for tumoral resection, and it was decided that no further imaging, such as cardiac magnetic resonance images (MRI) or coronary angiography, were needed. Considering the lack of guidelines regarding this clinical entity, the management of the case is mainly lead by the symptoms tied to the mass effect of the tumor. The presence of dyspnea as early as the lowest level of effort, required surgical removal of the pericardial mass. During the surgery, the incision was median sternotomy; the thymus and pericardial fat pad were found to be normal. After the pericardiotomy, it was noted that a soft, encapsulated epicardial tumor did not adhere to the pericardium and had a stalk measuring approximately $5 \mathrm{~cm}$ in diameter, attached to the left atrial roof (Figure 2). The pedicle was extending posteriorly toward the left atrioventricular groove. The amount of traction needed to expose the entire origin of the tumor was unsafe, and it was impossible to dissect without CPB support. The aorta and right appendage were cannulated so that the heart could be drained and safely mobilized. The entire mass measuring $12 / 8 / 5 \mathrm{~cm}$ was removed with $\mathrm{CPB}$ on beating heart using electric coagulation, (Figure 3 ) and $\mathrm{CPB}$ was easily weaned. The patient quickly was extubated, and his condition was uneventful in the intensive care unit. Histopathologic examination revealed the presence of diffuse proliferation of large and medium-sized mature adipocytes consistent with the diagnosis of pericardial lipoma (Figure 4). The patient was discharged from the hospital on the sixth postoperative day. The patient currently is asymptomatic with complete resolution of dyspnea with a marked improvement of his clinical state and effort tolerance and has not presented with evidence of recurrence at the 1-year follow up. 


\section{DISCUSSION}

Various lipomatous tumors may be found around the heart. Some of these lesions are discovered and have no consequence; others may require additional evaluation and management. CL can occur at any age and affect both genders equally. Typically, CL includes the right atrium and the left ventricle location, and they have origin either from the subendocardial (approximately 50\%), subpericardial (25\%), or from the myocardial layer (25\%) [Fine 1968]. Most CLs are asymptomatic, as the slow growth often delays diagnosis. When present, symptoms are variable, depending on the location or invasion of the underlying structures. The clinical findings are nonspecific and include dyspnea, fatigue, angina, palpitations, arrhythmias, atrioventricular block, precordialgia, syncope, left or right heart failure, pericardial constriction, interference in the electrical conduction system and even sudden death [Heath 1968; Mourad 2009; Valencia 2011; Noly 2016].

TTE, CT, and cardiac MRI are imaging methods allowing an analysis of the relationship between tumoral mass and adjacent structures and play a significant role in establishing a diagnosis. Attention should be focused on the localization of these lesions and extent of the disease, including invasion of adjacent structures, involvement of the coronary arteries, compression of cardiac chambers and pericardial effusion [Noly 2016]. Cardiac MRI also provides information about tissue composition [Dorobantu 2011] (difference with liposarcoma) and hemodynamic significance [Noly 2016]. In some occasions, it allows full characterization of tumoral mass features, hypodense tissue with attenuation of 20 to $50 \mathrm{HU}$ on CT images being characteristic for fat tissues [Steger 2011]. In the reported case, an intrathoracic giant lipomatous tumor was diagnosed from these imagery findings, but the origin from either the thymus, pericardial fat pad, or epicardial heart could not be determined. Although rare, there is a consensus that surgical resection should be attempted in all symptomatic patients. Total resection is preferred for encapsulated tumors to prevent local recurrence, although some authors consider that the rate of recurrence of subtotal resection of cardiac lipoma is quite low [Steger 2011]. In asymptomatic patients with incidentally small tumors, close monitoring is prudent. Surgical intervention may be considered, depending on the appearance of symptoms. Lipomas should be differentiated from liposarcomas not only by cardiac MRI features, but also histologically. CLs are made up of mature adipocytes that are limited by a collagenous capsule, while liposarcomas predominately are made up of mature fat cells [Steger 2011; Da Silva 2017].
CONCLUSION

CL originating from the left atrial roof is a very uncommon situation. It can be diagnosed at any age, regardless of gender, and can be associated with a wide range of symptoms that depend on the size, invasion of adjacent structures, and cardiac location. The first diagnostic tool for cardiac lipoma, even in asymptomatic cases, remains TTE. However, CT and MRI provide a better image, allowing the surgeon to evaluate more accurately the size, composition, and invasion of adjacent structures. However, these sometimes fail to determine the tumor's site of origin. Surgical resection is mandatory for symptomatic CL, but should be performed only in cardiac centers, with the use of $\mathrm{CPB}$ in posterior localizations.

\section{REFERENCES}

Da Silva, De Campos AMF, Vieira Baldo RC, et al. 2017. Pericardial lipoma: Diagnosed unexpectedly during heart failure investigation - Case Report. Curr Res Cardiol 4(4):55-57.

Dorobantu LF, Stiru O, Prodea A, et al. 2011. Unique case of primary malignant fibrous histiocytoma of the right ventricle with moderator band involvement. Heart Surg Forum 14(4):E245-E248.

Fine G. 1968. Neoplasms of the pericardium and heart. In: Gould SE, editor. Pathology of the Heart and Blood Vessels, 3rd. Springfield: Charles C. Thomas. 865 .

Heath D. 1968. Pathology of cardiac tumors The American Journal of Cardiology 21(3):315-327.

Iliescu VA, Dorobantu LF, Stiru O, et al. 2008. Second recurrence of cardiac myxoma 7 years after the initial operation. Chirurgia 103(2):239-241.

Italiano A, Ebran N, Attias R, et al. 2008. NFIB rearrangement in superficial, retroperitoneal, and colonic lipomas with aberrations involving chromosome band 9p22. Genes Chromosomes Cancer 47(11):971-7.

McAllister, Hall RJ, Cooley DA. 1999. Tumors of the heart and pericardium. Curr Probl Cardiol 24(2):57-116.

McAllister HA, Fenoglio JJ. 1978. Tumors of the cardiovascular system atlas of tumor pathology. 2nd series. Fascicle 15. Washington DC: Armed Forces Institute of Pathology 52-8.

Mourad OMA, Andrade FM, Abrahao P. et al. 2009. Massa mediastinal gigante assintomática: um raro caso de timolipoma. J Bras Pneumol 35(10):1049-52.

Noly PE, Mongeon FP, Rochon A, et al. 2016. Pericardial Constriction Caused by a Giant Lipoma Circulation 133:1709-1712.

Steger C. M. 2011. Intrapericardial giant lipoma displacing the heart. ISRN cardiology. 243637.

Valencia A, Lombo M, Correa J. 2011. Lipoma intrapericárdico gigante silente. Rev Colomb Cir 26:222-5. 